\title{
Cardiovascular Magnetic Resonance Imaging in Experimental Models
}

\author{
Anthony N. Price ${ }^{1, *}$, King K. Cheung ${ }^{1,2}$, Jon O. Cleary ${ }^{1,2}$, Adrienne E. Campbell ${ }^{1,2}$, \\ Johannes Riegler ${ }^{1,3}$ and Mark F. Lythgoe ${ }^{1}$ \\ ${ }^{I}$ UCL Centre for Advanced Biomedical Imaging, Department of Medicine and UCL Institute of Child Health, University \\ College London, UK \\ ${ }^{2}$ Department of Medical Physics and Bioengineering, University College London, UK \\ ${ }^{3}$ Centre for Mathematics and Physics in the Life Sciences and Experimental Biology (CoMPLEX), University College \\ London, UK
}

\begin{abstract}
Cardiovascular magnetic resonance (CMR) imaging is the modality of choice for clinical studies of the heart and vasculature, offering detailed images of both structure and function with high temporal resolution.

Small animals are increasingly used for genetic and translational research, in conjunction with models of common pathologies such as myocardial infarction. In all cases, effective methods for characterising a wide range of functional and anatomical parameters are crucial for robust studies.

CMR is the gold-standard for the non-invasive examination of these models, although physiological differences, such as rapid heart rate, make this a greater challenge than conventional clinical imaging. However, with the help of specialised magnetic resonance (MR) systems, novel gating strategies and optimised pulse sequences, high-quality images can be obtained in these animals despite their small size.

In this review, we provide an overview of the principal CMR techniques for small animals for example cine, angiography and perfusion imaging, which can provide measures such as ejection fraction, vessel anatomy and local blood flow, respectively. In combination with MR contrast agents, regional dysfunction in the heart can also be identified and assessed. We also discuss optimal methods for analysing CMR data, particularly the use of semi-automated tools for parameter measurement to reduce analysis time. Finally, we describe current and emerging methods for imaging the developing heart, aiding characterisation of congenital cardiovascular defects.
\end{abstract}

Advanced small animal CMR now offers an unparalleled range of cardiovascular assessments. Employing these methods should allow new insights into the structural, functional and molecular basis of the cardiovascular system.

Keywords: Cardiac MRI, mouse, rat, cardiac phenotyping, experimental models.

\section{INTRODUCTION}

Cardiovascular magnetic resonance (CMR) imaging has emerged as one of the most valuable tools for the diagnosis of heart disease. CMR is the only modality able to assess cardiac anatomy, function, viability, perfusion and metabolism within a single non-invasive examination $[1,2]$. Improvements in magnetic resonance (MR) scanner performance and hardware have rapidly increased the reliability and accuracy of clinical CMR. More recently, the introduction of parallel imaging and dynamic undersampling techniques has led to substantial accelerations in image acquisition to the extent that real time CMR is now possible. Such technology has opened up the opportunity to use magnetic resonance imaging (MRI) to guide interventional surgical procedures, in addition to further improving the spatial and temporal coverage in the most demanding techniques like first pass perfusion imaging.

*Address correspondence to this author at the UCL Centre for Advanced Biomedical Imaging Paul O'Gorman Building 72 Huntley Street London WC1E 6DD United Kingdom; Tel: +44-207-679-6295;

Fax: +44-207-905-2358; E-mail: a.price@ ich.ucl.ac.uk
CMR in small animals, however, still remains a significant technical challenge, primarily due to the spatial and temporal requirements. Applying MRI to a mouse heart, which can be less than one centimetre in length and beating in excess of 600 times per minute, requires specialist expertise and equipment. Nevertheless, CMR still offers the most accurate means to measure cardiac function and provide unrivalled images of heart anatomy in vivo, allowing for serial non-invasive assessment of animals in cardiovascular research [3, 4]. In this review we aim to provide an overview of the current CMR techniques routinely applied to experimental models of cardiovascular disease in adult mice and rats, and additionally give an insight to some of the emerging techniques that may enable a greater range of possible CMR studies.

\section{TECHNICAL CONSIDERATIONS}

\subsection{MR Acquisition and Hardware}

CMR has transformed the way cardiac function can be assessed in small animals, where left ventricular (LV) structure, function, perfusion and viability can all be evaluated within one scanning session, while maintaining a high de- 
gree of accuracy and avoiding the harmful effects of ionising radiation and toxic contrast agents. Most cardiac MRI experiments in small animals are performed using dedicated research scanners with a relatively small bore size (typically $20-30 \mathrm{~cm}$ ) compared to clinical systems. The strength of the magnetic field used in MR scanners determines the amount of signal available from a given scan duration and for this reason field strengths of between 4.7 and 9.4T are commonly used for imaging small animals [5], although systems of up to 21.1T are now being installed [6]. High-field scanners are also equipped with high performance gradient systems which are needed to achieve the necessary image resolution. Nevertheless, small animal imaging has been successfully performed on standard clinical systems using dedicated radio frequency (RF) coils, but inevitably the images produced do suffer from reduced signal associated with lower field strengths [7].

\subsection{Physiological Monitoring and Gating}

Unlike MRI of other anatomy, where the target organs are usually (or close to) stationary, the rapid movement of a beating heart poses a particular challenge in cardiac imaging. Motion is a fundamental vulnerability of MRI, and typically manifests as a ghosting artefact which can severely degrade image quality. While the normal range of resting heart rate in humans is $60-100$ beats per minute $(\mathrm{bpm})$, it can reach in excess of $600 \mathrm{bpm}$ in small animals. Therefore, a technique for compensating the effect of cardiac motion is essential for successful cardiac MRI. Prospective electrocardiogram (ECG)-gating is one of the most established techniques used in both human and small animals. The principle of ECG gating is to use the ECG waveform, typically the R-wave peak, to trigger data acquisition, and thus synchronising images to a particular stage in the cardiac cycle. The result is an image of a "frozen" heart, built up from many cardiac cycles. The validity of this technique is therefore limited by the assumption that there is negligible variation between heartbeats, which is true in most cases except in arrhythmias. Although the use of ECG-gating is well-established clinically, and can produce excellent results, specialised ECG equipment is required for use in small animals. These systems are designed to cope with the rapid heart rates, smaller ECG signals and increased electrical interference generated by the large gradient switching required to produce high resolution images.

Respiration is another source of undesired motion; the thoracic cavity and mediastinal organs such as the heart are displaced significantly during respiration. One solution is the use of MR navigator-echoes acquired during image acquisition to track the position of the diaphragm during respiration, allowing the subject to breathe freely throughout. However, with small animals it is most common to track respiratory movements via a neonatal apnoea pressure sensor placed over the chest or an inductive loop [8]. The combined cardiac and respiratory signals provide the essential gating information needed to synchronise image acquisition and to also provide remote physiological monitoring of anaesthetised animals within the scanner.

\subsection{Blood Flow Effects}

Even with careful gating strategies, images can be degraded by fast flowing ventricular blood at certain parts of the cardiac cycle. Images can suffer from local signal losses or even more detrimental flow artefacts depending upon the speed of data acquisition. Fast MRI acquisition sequences, such as spoiled gradient echo (GRE), are required to image the heart with so-called bright blood contrast (as shown in Fig. 1). An alternative way of suppressing flow-induced artefact is black-blood imaging, where the blood appears dark, which typically involves a double inversion preparation pulse prior to acquisition. This technique is particularly useful for imaging structural abnormalities of the ventricles and pericardium.

\subsection{Pulse Sequences}

In clinical cardiac MRI nearly all anatomical and functional cardiac imaging is performed using either a segmented fast spoiled GRE sequence, or more recently, the steady-state free precession (SSFP) technique has become the gold standard. Segmented spoiled GRE images are primarily $\mathrm{T}_{1^{-}}$ weighted and as a result the bright blood signal depends on fresh unsaturated (i.e. as yet unperturbed by RF energy) blood flowing through-plane to provide the contrast with the more saturated myocardium. SSFP on the other hand is somewhat independent to blood flow with image contrast dependent on the ratio $T_{2} / T_{1}$ and as a result provides excellent blood-myocardium contrast even with slow moving blood [9]. In addition to the improved contrast, SSFP is also one of the most signal to noise ratio (SNR) efficient sequences, providing greater signal in less time than spoiled GRE images. Unfortunately, SSFP images suffer from banding artefacts which are related to how short the repetition time can be made and the homogeneity of the magnetic field. As field inhomogeneity is directly related to field strength, it is extremely difficult to use SSFP for small animals at high fields. Thus, the primary sequence for assessing cardiac function in small animals is the original spoiled GRE used in the early days of human cardiac imaging. Following R-wave peak detection, a set number of echoes or k-space lines of data are acquired to make up the multiple frames of a cine image set. The process is repeated for many heart beats, typically 128 or 256 times, until all the data needed to reconstruct a full cine image stack are acquired, creating a movie (or cine) of the beating heart. Standard image orientations of the heart consisting typically of a 2- and 4-chamber long axis view and, in order to calculate global function, a complete stack (from apex to base) of short axis images are acquired (see Fig. 1).

\subsection{Image Analysis}

For almost all the animal models for which CMR is used, data analysis will be necessary to calculate functional parameters of the heart. Such parameters may be estimated for comparison at a single time point or for repeated measurements in order to study disease progression or treatment response. A typical CMR study will assess the standard global functional and morphological parameters of the heart, including ejection fraction (EF), end-diastolic volume (EDV), end-systolic volume (ESV), stroke volume (SV), and left ventricular mass (LVM). Additional parameters such as myocardial perfusion, infarct size, myocardial strain, peak ejection and filling rates or the effect of agents such as dobutamine may also be assessed. 

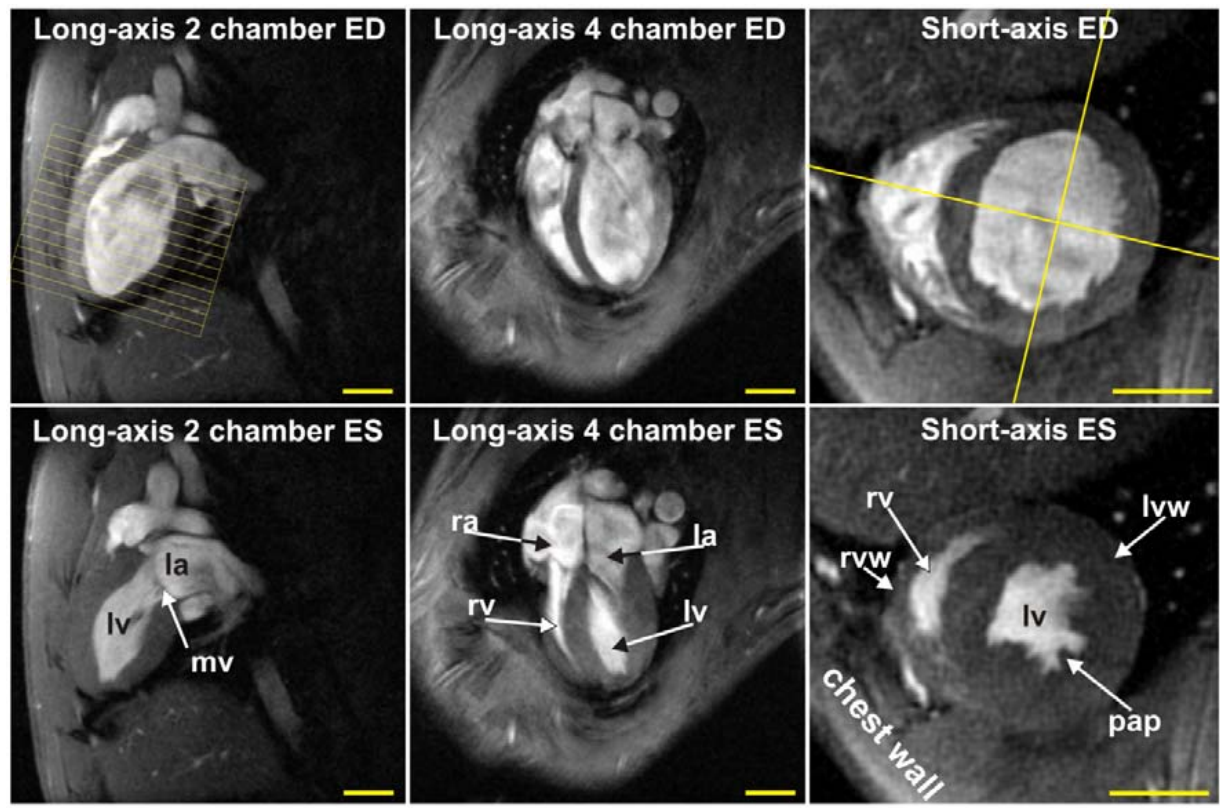

Fig. (1). Example of bright blood cine images of a rat heart at 9.4T. Standard orientations consisting of two and four chamber long-axis and short-axis views are acquired. The upper row shows corresponding images for end diastolic (ED) time frame and the lower row end systolic (ES) time point. la: left atrium, mv: mitral valve, lv: left ventricle, ra; right atrium, rv: right ventricle, lvw: left ventricular wall, rvw: right ventricular wall, pap: papillary muscle. The scale bar represents $1 \mathrm{~cm}$.

Central to any CMR study is the analysis of cine data for the standard functional parameters. These are commonly calculated from a series of cine images taken from the apex to the base of the heart, which require accurate detection of the epicardial and endocardial borders (see Fig. 2). A clear set of rules for the inclusion of slices, maximum heart rate variability between slices, the handling of papillary muscles and the definition of the valve planes should be adapted to ensure accuracy, as well as low inter- and intra-observer variability. In addition, data should always be randomised and blinded before analysis to avoid observer bias.

In many published studies, papillary muscles are often not segmented as myocardium and treated as part of the blood pool. However, it can be argued that papillary muscles should be segmented as myocardium since they account for a sizable fraction of the blood-pool volume at end-diastole (up to $10 \%$ ) [10]. This approach is commonly adopted due to its simplicity, as the geometry of the blood pool is made much simpler (i.e. circular) by omitting the papillary muscles, thus making segmentation and measurement more time efficient. Nevertheless, the potential effects of omitting the papillary muscles on measurement accuracy should be considered during analysis, especially with estimation of ESV in which the LV blood-pool volume is lowest.

Manual segmentation of CMR data is commonly used and yields low inter- and intra-observer variability $[3,11]$. The accuracy of heart volume measurements via MRI has been validated by comparing calculated myocardial mass and ex vivo measurements, showing a high correlation between both methods $[3,12,13]$.

The increasing demand for CMR and the time consuming nature of manual segmentation has lead to development of commercially available semi-automatic segmentation methods. These may offer a considerable reduction in analysis time while showing similar inter- and intra-observer variability $[11,14]$. Such segmentation programs use different approaches from simple thresholding to more advanced deformable models for volume estimation and generally include long axis views to improve valve definition accuracy.

\section{TECHNIQUES FOR ASSESSING CARDIAC PHYSIOLOGY}

\subsection{Myocardial Mass}

Myocardial mass, or left ventricular (LV) mass in particular, is an important index for the assessment of cardiac functional status for a variety of clinical conditions such as cardiomyopathy, hypertension, and valvular disease. MRI can be used to quantify myocardial mass by acquiring a volume dataset of the heart. A delineation of the epicardial and endocardial contours of the left ventricle is performed, after which the segmented volume is multiplied by the myocardial specific gravity $\left(1.05 \mathrm{~g} / \mathrm{cm}^{3}\right)$ to calculate the $\mathrm{LV}$ mass. Accurate in vivo quantification of myocardial mass using MRI has been demonstrated and validated in the mouse and rat $[4,15$, 16]. Clinically, MRI has been shown to be more accurate and reproducible than M-mode, 2D and 3D echocardiography [17-19].

\subsection{Wall Motion}

Global and local myocardial motion can be assessed by myocardial tagging strain analysis, using sequences such as spatial modulation of magnetisation (SPAMM) or phase contrast MRI [20]. In SPAMM, spatially selective RF pulses are applied to pre-saturate thin planes of myocardium perpendicular to the imaging plane at end-diastole (see Fig. 3), which are then used as markers for regional myocardial movements $[21,22]$. MRI tagging can be applied in two or three dimen- 


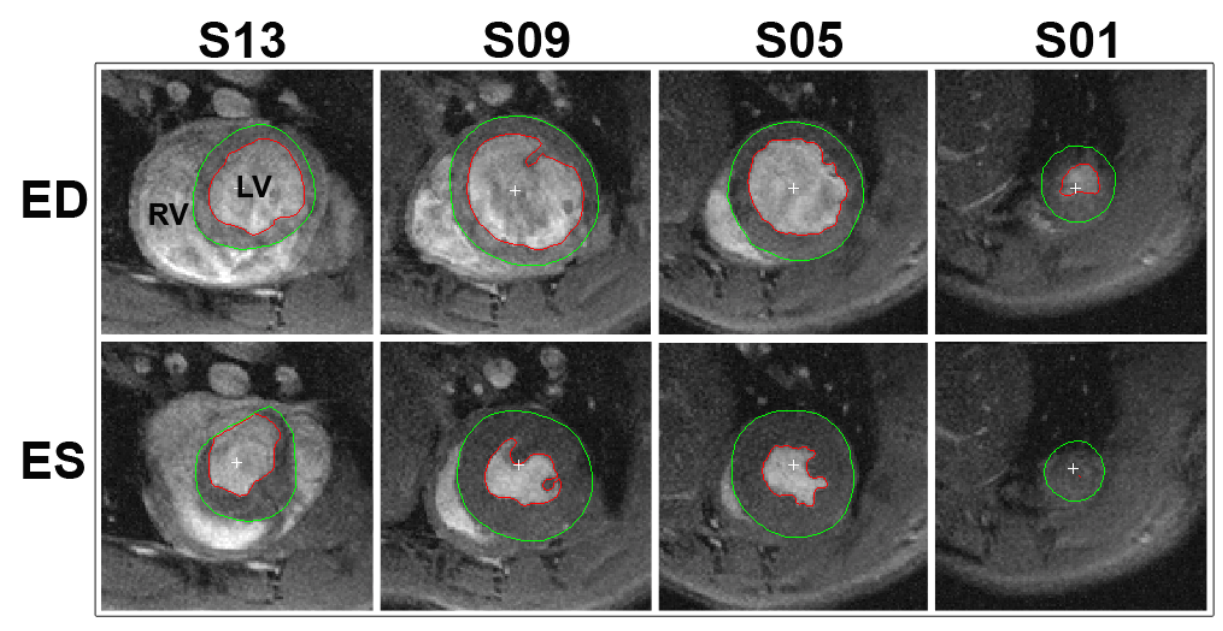

Fig. (2). Segmentation of a typical cine stack of a rat heart. The red line delineates the endocardial border, whilst the green line marks the epicardial border. The top and bottom rows depict the end diastolic (ED) and end systolic (ES) time frames, respectively. The columns show different slices where the most apical slice included is labelled as slice 1 (S01). Parameters; in-plane resolution: $200 x 200 \mu \mathrm{m}$, slice thickness $1 \mathrm{~mm}, \mathrm{RV}$ : right ventricle, LV: left ventricle.

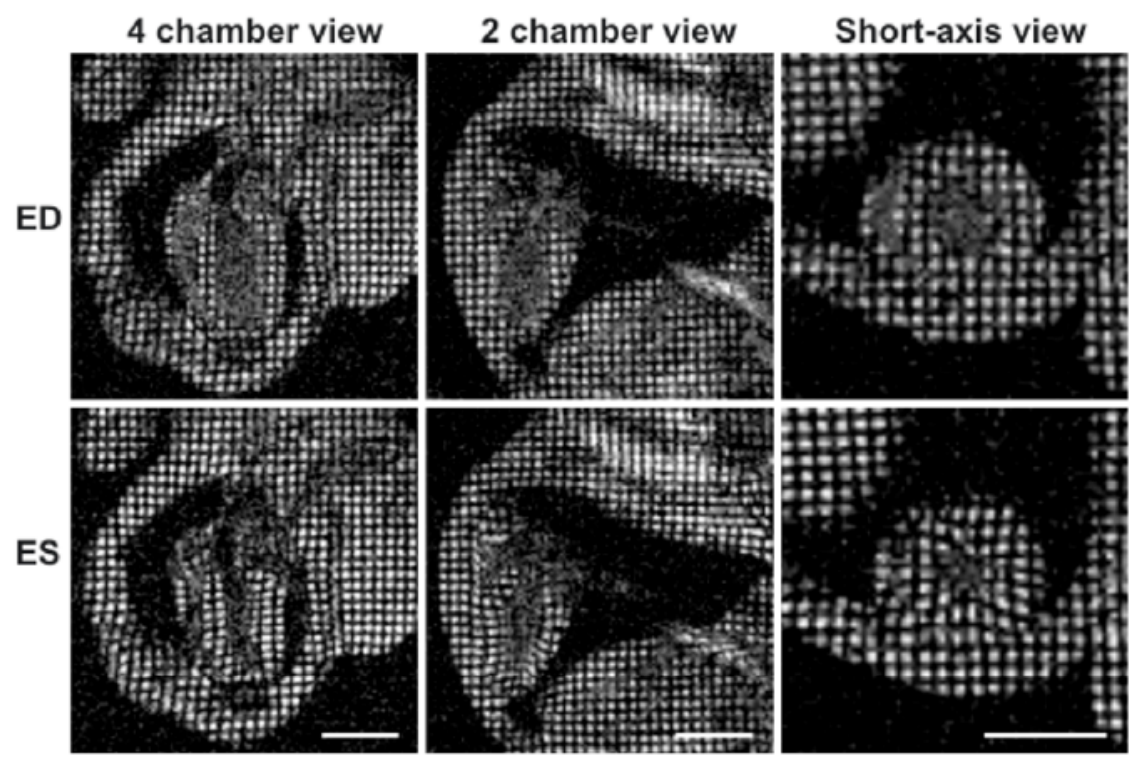

Fig. (3). Myocardial tagging in the mouse. Four chamber, two chamber and short-axis views of a mouse heart at end diastole are shown in the top row, while end systolic frames are depicted in the lower row. The movement of the tagged myocardial tissue can be tracked to calculate myocardial strain. The scale bar represents $5 \mathrm{~mm}$.

sions to assess radial, circumferential and longitudinal shortening $[23,24]$. The pre-saturated thin planes, or tags, appear dark on the image and persist throughout a cardiac cycle. During myocardial contraction, these tags are displaced in accordance to their underlying myocardial motion. The degrees of displacement are then used to evaluate myocardial contractility using tag detection and displacement-estimation algorithms [25, 26]. Myocardial displacement is calculated and reconstructed in three dimensions to provide detailed strain maps of the tagged myocardium in relation to time and space [24]. Although an accurate assessment of three-dimensional LV function can be obtained with this technique, data analysis is slow and can require significant user input [27].

Faster data processing and image acquisition techniques such as; harmonic phase, displacement encoding with stimu- lated echoes [28], and fast strain-encoded MRI [29] are emerging. Harmonic phase MRI utilises the phase component of images acquired with SPAMM, which contains an array of harmonic (or spectral) peaks in the Fourier domain. A single off-centred peak contains motion information, where the phase of a complex image (inverse Fourier transform) of such a peak is linearly related to the true motion [30]. By isolating these peaks, the motion of the underlying tissue could be tracked automatically with great accuracy [31].

Myocardial tagging has been validated in a canine model with sonomicrometry [32], and was used to evaluate myocardial strain during local ischaemia in dogs [30, 33] and after myocardial infarction in rats [27]. It has also been applied to characterise regional myocardial function in the 
mouse [23] and to demonstrate that left ventricular torsion is equal in mice and humans [34].

\subsection{Perfusion}

Perfusion measurement plays an important role in early detection of occlusive coronary heart disease, as well as determining the severity of occlusion and viability of myocardium [35]. A characteristic feature of occlusive coronary heart disease is the reduction in myocardial flow reserve, which can be detected before clinical symptoms or LV dysfunction become evident [36]. Currently, there are two main techniques for perfusion studies: Arterial spin labelling (ASL) and contrast-enhanced first-pass perfusion imaging.

The first-pass perfusion technique tracks the transit of blood by using gadolinium-based contrast agents; gadolinium is paramagnetic and shortens $\mathrm{T}_{1}$ relaxation, thus giving higher signal intensity in $\mathrm{T}_{1}$-weighted images. During a firstpass perfusion scan contrast agent is injected peripherally, which then passes through the venous system into the heart chambers. A fast (single-shot) $\mathrm{T}_{1}$-weighted sequence is used to image the myocardium as the contrast agent reaches the coronary arteries and perfuses through, with enhancement peaking approximately 10 heartbeats after passing the LV chamber [37]. Signal intensity correlates with contrast concentration, and hypo-enhanced areas indicate reduced or no tissue perfusion. The technique is well-established clinically and is the most practical method for evaluating myocardial perfusion [37], however it remains a significant challenge for use in small animals. In order to capture the arrival of the first-pass of contrast agent, complete image frames need to be acquired in a relatively motion-free portion of the cardiac cycle, so for mice this could be in the order of 10-30 ms. In addition, reproducible bolus injections are hard to achieve in small animals, making accurate quantitative perfusion studies extremely difficult to perform $[5,38]$.

Alternatively, arterial spin labeling (ASL) may be used to non-invasively quantify perfusion. ASL perfusion measurements are generated by the comparison of two $\mathrm{T}_{1}$ maps: i) using a global inversion recovery and ii) with a sliceselective inversion recovery for a slab slightly larger than the imaging slice. In the slice-selective case, spins (proton signal) that enter the imaging slice due to perfusion are not inverted, causing an apparent acceleration in $\mathrm{T}_{1}$ recovery. An assessment of the difference between the global and sliceselective $T_{1}$ maps provides a quantification of the blood perfusion in the imaging slice. Fig. (4) displays $T_{1}$ maps gener- ated by global and slice-selective inversion and a perfusion map for a mouse at baseline under general anaesthesia.

This technique has been adapted for use in the myocardium of mice and rats in a limited number of studies (sequences are presented in [39-41]). Blood flow values have been found to be comparable with microsphere measurements [41-43], although low SNR and speed are the main limitation of this technique. To date, ASL has been used to assess perfusion in a diverse range of applications, including myocardial infarction models [41], nitric oxide synthase knockout mice [44], dobutamine stress tests [40], vasodilatation tests and effects of a variety of anaesthetics [44-46].

Another technique, widely used in functional neuroimaging, is blood oxygen level dependent (BOLD) imaging, and has been applied to assess myocardial perfusion [47]. Traditional BOLD imaging is based on $\mathrm{T}_{2}{ }^{*}$-weighted sequences as previous research in the brain has shown $\mathrm{T}_{2} *$ to be more sensitive than $T_{2}$ to changes in oxygen saturation [48]. However, $\mathrm{T}_{2}$ *-based BOLD cardiac imaging poses significant problems due to local field inhomogeneities, exacerbated by the susceptibility of a GRE sequence with long echo times [49]. Alternatively, $\mathrm{T}_{2}$ could be used, but is less sensitive to oxygen saturation changes. By using a $\mathrm{T}_{2}$-prepared SSFP BOLD sequence, Shea et al. demonstrated a good correlation between changes in signal intensity and myocardial blood flow [49].

\subsection{Viability}

Myocardial viability is defined as the presence of living (or viable) myocytes, and is an important index in assessing the extent of post-ischaemic injury. It is important to note that a portion of myocytes might have impaired contractility after an ischaemic assault, possibly due to reduced perfusion, but are still alive. These cells are known as hibernating myocytes and their functionality is potentially fully recoverable when normal myocardial blood flow is restored. Two frequently used clinical MRI techniques, dobutamine stress and late gadolinium enhancement, offer the means to differentiate between hibernating and non-viable myocytes.

In dobutamine stress MRI, a series of cine images are acquired while increasing doses of dobutamine, an inotropic agent, are infused into the subject (see Fig. 5). Any akinetic or dyskinetic myocardial segments that are viable (ie. contain hibernating myocytes) would show an improvement in contractility after administration of dobutamine. This tech-
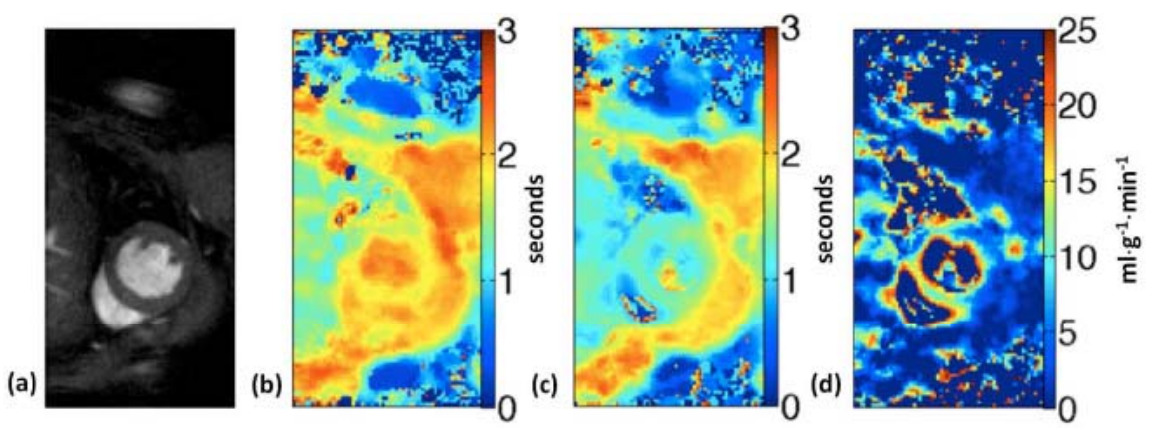

Fig. (4). ASL perfusion imaging in the mouse. Short-axis image (a), $\mathrm{T}_{1}$ maps from global inversion (b) and slice-selective inversion (c) are used to generate the perfusion map (d) obtained from a mouse at baseline. 

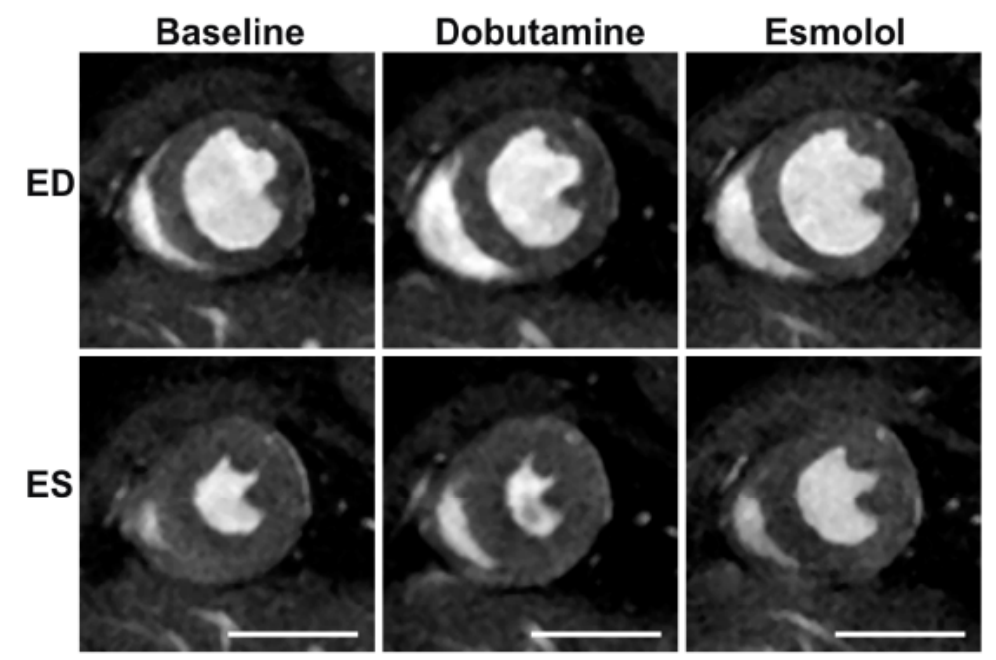

Fig. (5). Assessment of pharmacological agents on cardiac function in a normal mouse. Baseline images (first column) acquired prior to an infusion of dobutamine (middle column). In addition, the effect of the beta blocker esmolol (right column) is subsequently shown to counteract the increased contractility caused by the cardiac stress agent dobutamine. ED; end diastolic, ES; end systolic time frames. The scale bar represents $5 \mathrm{~mm}$.

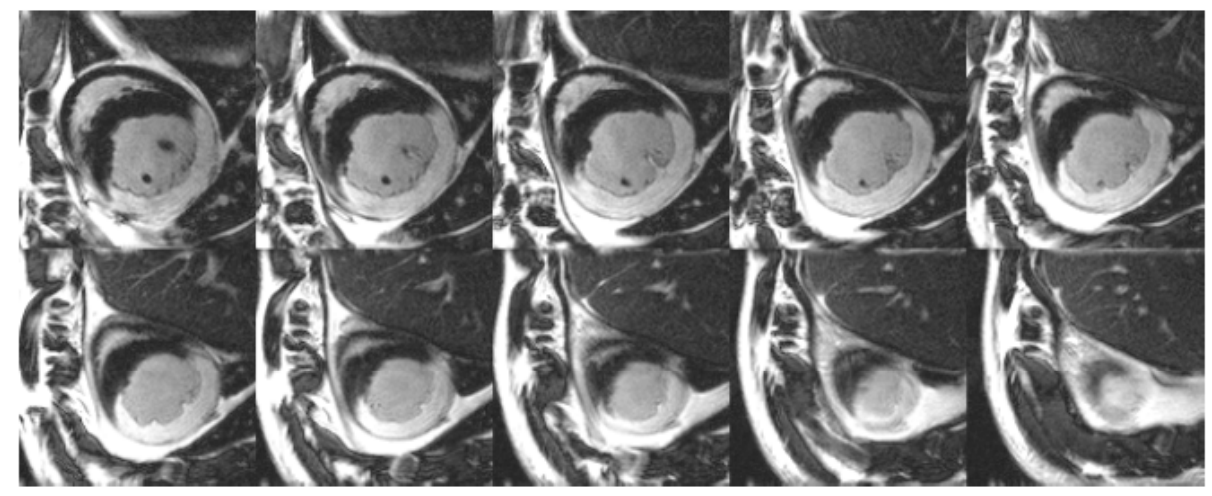

Fig. (6). Late gadolinium enhancement MRI in a rat 2 hours following surgery to induce myocardial infarction. A series of consecutive shortaxis images reveal a large area of non-viable myocardium extending down to the apex. Imaging was performed $\sim 10$ minutes following injection of Gd-DTPA. From reference [61].

nique has been shown to adequately predict improvement in LV function in patients after revascularisation [50], and was employed to investigate acute and chronic cardiac remodelling after MI in mice and rats [51, 52]. Myocardial tagging can be incorporated into dobutamine stress MRI and has demonstrated superior accuracy over studies without tagging [53], and produced similar accuracy to dobutamine stress echocardiography (DSE) [54]. Currently, there are still only a limited number of experimental studies that have utilised dobutamine stress MRI in small animals [16, 44, 51, 55].

The other approach for assessing myocardial viability is delayed enhancement MRI or late gadolinium enhancement (LGE) which involves administering a contrast agent to directly image non-viable myocardium (see Fig. 6). In this method, an extracellular contrast agent (usually Gd-DTPA) is injected into the subject. On first-pass images, normal myocardium with normal perfusion would be enhanced by the contrast agent, whereas infarcted regions would appear hypoenhanced due to reduced or absent perfusion. However, the hypoenhanced region would gradually become hyperenhanced as a consequence of increased retention of contrast agent, which is thought to be caused by increased extracellular matrix within collagenous scar, and delayed washout due to reduced capillary density [56]. This method is wellvalidated clinically for predicting the distribution of infarcted myocardium [57] and for excluding irreversible damage where no delayed enhancement is observed [58, 59]. It is also well-validated in a mouse occlusion/ reperfusion model with 2,3,5-Triphenyltetrazolium chloride staining [60], but results are varied in the rat model [5].

Manganese-enhanced MRI (MEMRI) is an alternative technique used in experimental cardiac studies. Similar to Gd agents, $\mathrm{Mn}^{2+}$ is also paramagnetic and primarily acts by reducing $T_{1}$ of surrounding tissue to cause signal enhancement on MR images. However, its strength in small animal studies is its use as a molecular agent, since $\mathrm{Mn}^{2+}$ ions have physical properties similar to calcium ions, and therefore taken up by excitable cells such as neurons and cardiomyocytes where $\mathrm{Ca}^{2+}$ influx readily occurs. In cardiomyocytes in particular, $\mathrm{Mn}^{2+}$ is able to enter the cell through voltagegated calcium channels. However, in contrast to $\mathrm{Ca}^{2+}, \mathrm{Mn}^{2+}$ is retained after uptake, which can lead to cardiotoxicity. 
The key use of MEMRI in cardiac imaging is to produce a signal enhancement on MRI that relates to cardiac function. In the mouse heart, MEMRI has been shown to be sensitive to inotropic changes [62]. Administering $\mathrm{Mn}^{2+}$ by intravenous infusion, forming a stable plasma concentration allows baseline signal intensity measurement to be taken. After administration of a inotropic agent, such as dobutamine, MRI signal can be seen to increase in more active areas of myocardium. Similarly, in animal models of myocardial infarction, $\mathrm{Mn}^{2+}$ may also be used to delineate between infarcted and healthy myocardium on the basis that uptake of $\mathrm{Mn}^{2+}$ is greater in regions of functioning tissue where viable cardiomyocytes are abundant.

\subsection{Angiography}

Magnetic resonance angiography (MRA) in small animals is most commonly performed using the time-of-flight (TOF) approach [63-65]. The technique relies on the same contrast mechanism utilised in bright blood imaging, but typically 3D images are collected from multiple acquisitions of overlapping thick slabs rather than 2D slices. Vascular anatomy can then be visualised with rendered maximum intensity projection 3D volumes (see Fig. 7). Most applications of small animal MRA have been limited to cerebrovascular anatomy, applying the technique to the vessels surrounding the heart is particularly challenging. However, there have been reports of using TOF MRA to visualise even the coronary arteries in the living mouse [66]. Low-doses of gadolinium contrast agents can be used to further enhance the TOF effect and improve image quality. They can be used to reduce the overall acquisition time which can be particularly long when cardiac and respiratory gating is used, which is essential for visualising the smaller vessels located close to the heart [67].

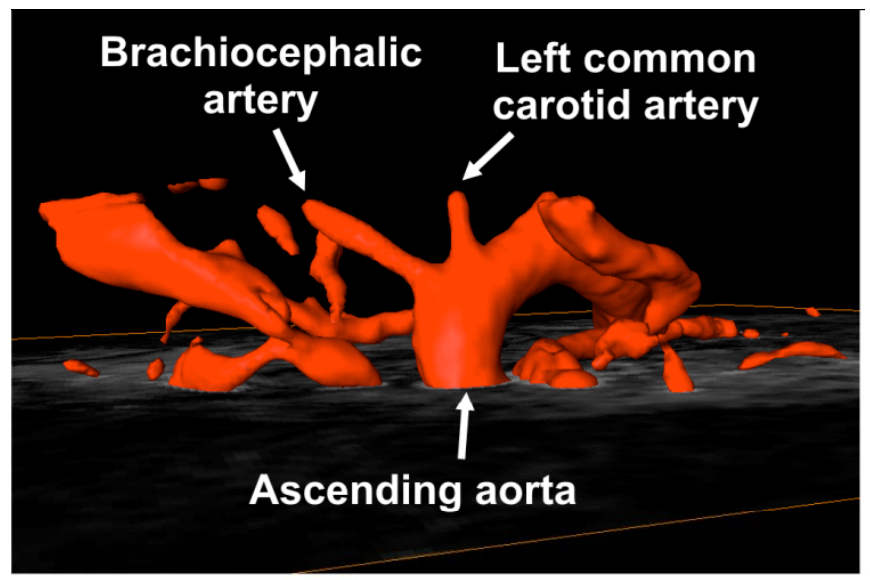

Fig. (7). In vivo magnetic resonance angiography of an adult mouse showing the major vessels superior to the base of the heart. TOF techniques provide high contrast between flowing blood and surrounding tissue, image data can be reconstructed using maximum intensity projection to give $3 \mathrm{D}$ renders of vessel geometry.

\subsection{Vessel Wall and Plaque Imaging}

Vessel wall imaging in small animals remains challenging due to the extremely high resolution required. Ohlstein et al [68] and Chandra et al [69] performed in vivo (at 4.7T) and ex vivo (at 9.4T) carotid artery imaging in rats. In these ex vivo studies, the various layers of the arterial wall were clearly defined. In vivo vessel wall imaging of the mouse common carotid artery was performed in another study [70] by using a composite image-reconstruction method, where $\mathrm{T}_{1}, \mathrm{~T}_{2}$ and $\mathrm{PD}$-weighted images were assigned a separate colour channel, and combined to form a 24-bit colour image. The resultant contrast made the vessel wall distinguishable, but not sufficient for accurate measurements. In another study, the mouse coronary arteries and the four valves were successfully imaged in vivo, without the aid of contrast agents [66]. Vessel wall and plaque imaging have also been performed in other knockout mouse models [71-74]. Targeted contrast agents like SPIOs (super-paramagnetic iron oxide particles) conjugated to antibodies can be used to image specific molecular processes involved in disease progression [75].

\subsection{Myocardial Micro- and Macro-Structure}

Diffusion tensor imaging (DTI) has the potential of characterising the remodelling of cardiac fibre structure after infarction in rats, and is based on the theory that water diffusion will be the greatest parallel to the myocardial fibre orientation, and therefore the primary eigenvector of the diffusion tensor coincides with the local fibre orientation. By comparing DTI and conventional histological results, recent studies using dogs, goats and rabbits [76-78] have demonstrated a good correlation between MRI and histological fibre angle measurements, thereby validating the DTI approach for complete characterisation of myofibre architecture. MR-microscopy and diffusion imaging have also been performed on isolated and fixed rat hearts [79-82]. DTI may potentially be a valuable tool for defining structural remodelling in diseased myocardium at cellular and tissue levels.

\subsection{Metabolic Characterisation}

MR spectroscopy is a technique which can noninvasively measure the relative concentrations of molecules. It can be used to characterise the concentrations of high energy phosphorous compounds involved in cardiac energy metabolism for a selected region of the myocardium. Phosphorus molecules of interest are adenosine triphosphate (ATP), phosphocreatine (PCr) and inorganic phosphates (Pi). Myocardial ischaemia leads to a significant reduction in the $\mathrm{PCr} / \mathrm{Pi}$ ratio, as well as a reduction in the $\mathrm{PCr} / \mathrm{ATP}$ ratio. ${ }^{31} \mathrm{P}$ MR spectroscopy has been demonstrated to be feasible for measurements of the PCr/ATP ratio in isolated mice hearts $[83,84]$. Concentration of intracellular sodium is another indicator for myocardial ischaemia, and could be distinguished from extracellular sodium using ${ }^{23} \mathrm{Na}$ MRI with chemical shift imaging [85]. Cardiac metabolic imaging of other nuclear magnetic resonance-visible nuclei such as ${ }^{13} \mathrm{C}$ and ${ }^{19} \mathrm{~F}$ have also been investigated, but experience in this field is still limited $[86,87]$ and is particularly challenging to implement successfully.

\section{EXPERIMENTAL ANIMAL MODELS OF CARDIOVASCULAR DISEASE}

\subsection{Myocardial Ischaemia}

Myocardial ischaemia occurs when there is an imbalance between myocardial oxygen supply and consumption due to reduced coronary blood flow [88], which if not restored 
promptly would ultimately lead to infarction of myocardium. Clinically, myocardial ischaemia is most commonly caused by thrombotic occlusion of a coronary artery, which is initiated by the rupture of an atherosclerotic plaque. Since this is a spontaneous process, it is difficult to mimic; therefore myocardial ischaemia in most animal models is achieved by occluding the coronary arteries mechanically either by direct ligation or intraluminally, with the former being the most popular technique in small animals including mice and rats, and the latter method largely confined to bigger animals such as dogs and pigs [89]. With the direct ligation method, the animal is anaesthetised and ventilated, after which the heart is exposed typically via a left thoracotomy. The left anterior descending coronary artery (LAD), which supplies the anterolateral region of the left ventricle, is then ligated using sutures and a snare. Successful occlusion is evident by the rapid pallor over the anterolateral region of the left ventricle that is perfused by the LAD, as well as associated ECG changes.

The evolution of ischaemia to infarction and subsequent ventricular remodelling is characterised by the progression of injured myocardium to fibrosis and corresponding wall thinning. Compensatory hypertrophy of the remote healthy myocardium may follow, after which the infarcted heart may then progressively dilate and eventually undergo failure [90]. By using animal models, the site, duration and degree of coronary occlusion can be manipulated to alter the character- istics of the resulting ischaemia and infarction, and therefore allowing the different stages of myocardial infarction to be studied. Different variants of myocardial infarction models will be discussed below.

\subsubsection{Ischaemia without Reperfusion (Permanent Occlusion)}

In a permanent occlusion model, the LAD artery is ligated permanently with a silk suture and therefore the ischaemic myocardium is not reperfused. As a result, infarction is often more extensive and transmural in nature, and subsequent fibrosis and wall thinning is achieved more rapidly (see Fig. 8). This approach is thus valuable for investigating the later stages of myocardial infarction, such as ventricular remodelling and heart failure [91].

\subsubsection{Ischaemia with Reperfusion (Transient Occlusion)}

This approach involves a transient period of ischaemia followed by the release of ligation to allow reperfusion of the previously ischaemic myocardium (example shown in Fig. 9). Transient occlusion represents more closely the ischaemia-reperfusion scenario that is found clinically, where patients are revascularised following an acute ischaemic event. Therefore, the ischaemia-reperfusion model is popular amongst interventional studies, including those using statins [92] and stem cell therapy [93]. In addition, the ischaemia-
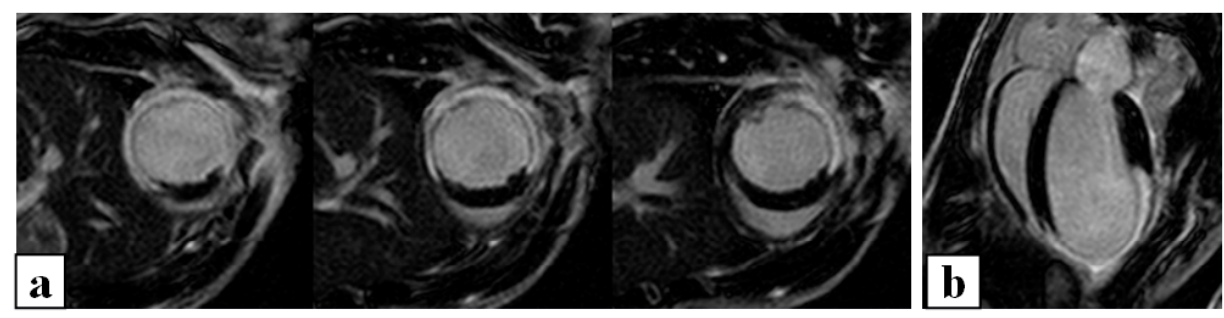

Fig. (8). Permanent occlusion model of myocardial ischaemia in the mouse. Late gadolinium images shown here are acquired $48 \mathrm{hrs}$ after myocardial infarction (MI) surgery with 3 consecutive short-axis slices (a), and a 4-chamber long-axis view (b), revealing myocardial ischaemic region and the early stages of wall thinning (figures from reference [61]).

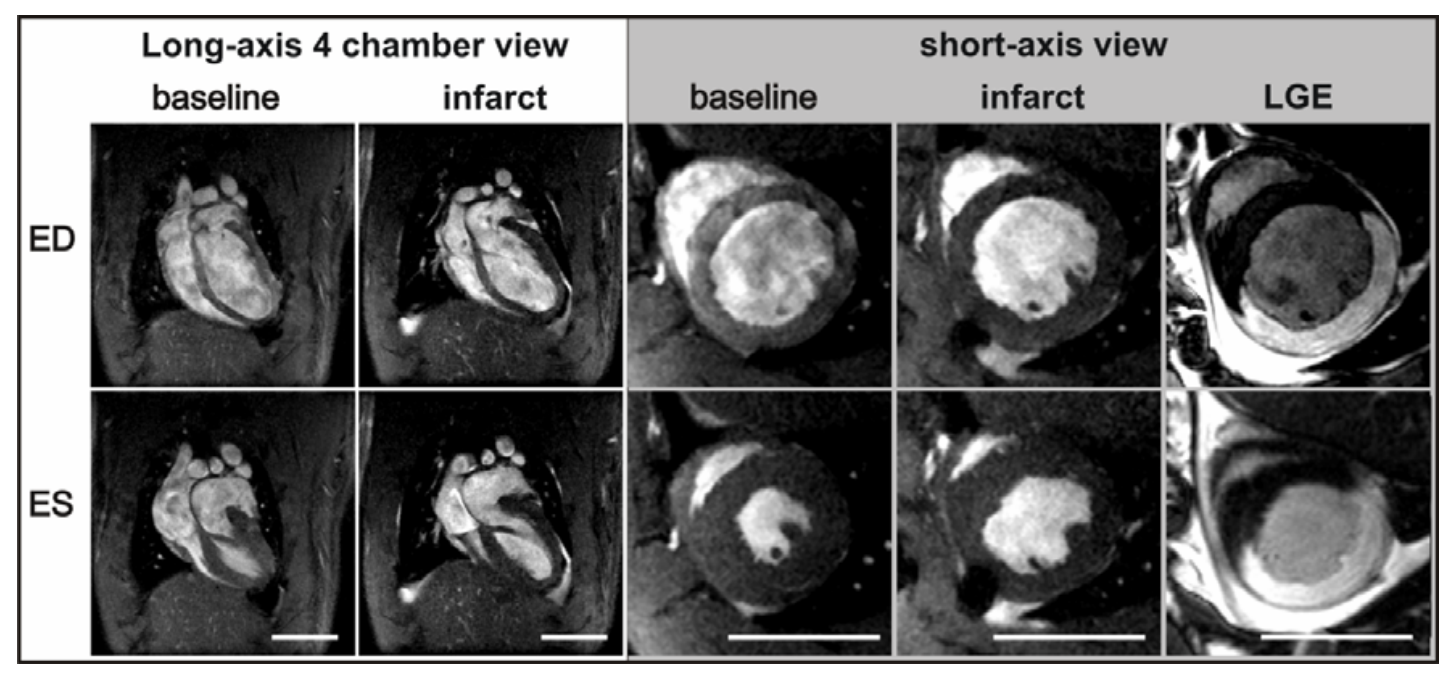

Fig. (9). Example of reperfused MI in a rat. Images of the infarcted heart were acquired two hours after reperfusion of the occluded left anterior descending coronary artery. The size of the infarcted myocardium can be roughly estimated via reduced contractility on short axis views. Late gadolinium enhancement (LGE) (far right) can be used for accurate assessment of the infarct size. The size changes associated with cell death are exploited with this method and can be seen as enhancement of the myocardium. ED; end diastolic and ES; end systolic time points. The scale bar represents $1 \mathrm{~cm}$. 
reperfusion model is also valuable for investigating reperfusion injury, a phenomenon believed to contribute to further myocardial damage following reperfusion [94].

\subsection{Atherosclerosis and Vascular Injury Models}

\subsubsection{Atherosclerosis Models}

Atherosclerosis, a precursor of myocardial infarction and stroke, is a common inflammatory disease of complex pathogenesis and multiple factors such as thrombosis, hyperlipidaemia and endothelial injury. It is characterised by the accumulation of lipids and fibrous components, the recruitment of monocytes and lymphocytes, and hyperplasia of media, all of which contributing to the subsequent thickening and reduced elasticity of the arterial wall [95]. The rabbit is one of the most investigated animals in atherosclerosis research, since they develop spontaneous or high-lipid diet-induced atherosclerotic plaques [96]. While rodents are naturally resistant to atherosclerosis, several transgenic mouse strains such as the apoprotein-E knockout have been developed to introduce lipid abnormalities and subsequently atherosclerosis [97]; such techniques offer the potential to study the role of isolated metabolic pathways in atherosclerosis.

The stability of an atherosclerotic plaque is an important factor, since vulnerable plaques - characterised by a thin cap and large lipid core - are prone to rupture, where the ensuing thrombosis is often the cause of coronary occlusion [98]. Therefore, the ability to differentiate between stable and vulnerable plaques is of great clinical relevance; however, there is currently no robust biomarker to distinguish between the two due to their similar composition and pathophysiology. Recently, imaging techniques such as intravascular ultrasound and high-resolution MRI have provided some promises in identifying vulnerable lesions in the clinical settings $[70,99]$.

\subsubsection{Endothelial Injury and Restenosis Models}

Localised arterial wall and endothelial injury can be produced by mechanical means to investigate restenosis (renarrowing of the vessel) in animal models. This disease is associated with transluminal angioplasty, a technique widely used for coronary revascularisation following myocardial infarction, where a small balloon is inserted and inflated to expand the occluded artery. The procedure invariably causes injury to arterial wall and in particular endothelial denudation, triggering cell proliferation, intimal thickening and ultimately restenosis that may require repeated treatment. In rats, the common carotid artery is often used to create endothelial injury due to its accessibility and size [100]. Assessment of injury and disease progression in this model have traditionally relied on histopathological analysis with crosssectional slides [101]; increasingly, standard MRI and MRA techniques as well as the more novel MR microscopy and contrast agents are being used to assess the vessel wall and quantify the degree of restenosis in addition to histology [68, 69, 102, 103].

\subsubsection{MR Assessment of Cellular Therapies}

The discovery of stem cells and their potential for tissue regeneration led to the hypothesis that regeneration of the adult damaged heart might be possible via stem cell transplantation. It is hoped that stem cells will differentiate into cardiomyocytes or fuse with resident myocytes and functionally integrate if they receive the right clues from their microenvironment. Following this hypothesis many studies in animals [104-112] and a few clinical trials [112-114] have been conducted. Some of the cell transplantation studies in small animals showed considerable improvement in cardiac function while others showed only mild improvements. However, these improvements could not be translated into human trials. It is, therefore, necessary to improve the animal models in order to derive clinically relevant information from them. These studies employed different analysis methods such as MRI, PET and echocardiography which is probably one of the sources for variability.

Another problem with small animal models is the quantification of the amount of cells and their specific localisation. MRI together with stem cells labelled with superparamagnetic iron oxide particles (SPIOs) can be used to address these issues. SPIOs generate local field inhomogeneities when placed into a magnetic field. This causes local hypointensity (darkness) in $T_{2}$ or $T_{2}{ }^{*}$ weighted MR images (see Fig. 10). The sensitivity of $\mathrm{T}_{2}{ }^{*}$ images to SPIOs allows the detection of a few thousand cells or even single cells [115-119]. Several publications indicate that the labelling of stem cells with small amounts of SPIOs does not alter their viability and differentiation potential [120-122]. However, there is always the possibility of functional changes. Therefore, a careful optimisation of the labelling strategy should be performed before it is used in vivo. SPIO labelled cells offer the possibility to confirm the delivery success of a particular strategy and allow the tracking of cell movement over time.

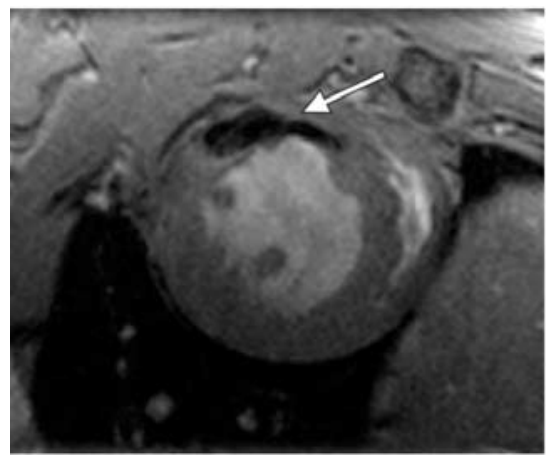

Fig. (10). Short axis view of a rat heart: mid-ventricular slice at end systolic time point. The arrow points to the hypointense region where stem cells were injected one week prior to image acquisiton into the border region of an infarct. $\mathrm{T}_{2}{ }^{*}$ weighted spoiled GRE sequence used.

The quantification of delivery success is important to establish if there is a dose dependent relationship between the amount of cells delivered and the functional outcome of a study. However, this has to be done with caution. Firstly, imaging artefacts which cause hypointense regions are frequently observed on cardiac cine images. Secondly, the hypointensity observed from SPIOs is only indirectly coupled to the cells administered in particular over a long time course. SPIOs are frequently taken up by neighbouring cells and macrophages when cells die. As long as those cells and macrophages stay in the same imaging voxel they cannot be differentiated from the transplanted cells. A major limitation of SPIO based cell tracking is the inability to assess cell 
viability. It might, therefore, be necessary to use an additional labelling strategy which allows assessment of cell viability over time. Depending on the method, it might allow for the combination of high spatial resolution MRI with in vivo cell viability assessment.

\section{IMAGING THE DEVELOPING HEART WITH MRI}

\subsection{Ex Vivo Morphological Phenotyping of Mouse Models}

Since the early part of the $20^{\text {th }}$ century, mice have been used widely for genetic investigations [123] and more recently methods have emerged to enable direct manipulation of the mouse genome. The mouse has become the key model for research into human disease. With the announcement of the completion of the first draft of the mouse genome in 2002 [124], a number of large-scale programmes are underway to systematically create transgenic knockout models [125]. The study of the embryos of such mouse mutants is useful to determine effects of genes on development and congenital disease. Given the commonality in molecular pathways across the embryo, insight may be obtained into novel genes involved in the basis of heart development. The study of the effect of genes on mouse embryo development is particularly relevant to the investigation of congenital heart defects (CHDs).

Morphological analysis of embryo anatomy is an important part of phenotype characterisation. Conventionally, this is done ex vivo by microscopic examination with histology. In this method, embryos are ethanol-dehydrated, embedded in paraffin wax and thinly sectioned into $2-8 \mu \mathrm{m}$ slices [126]. This provides high resolution $2 \mathrm{D}$ data and sections that may also be stained for gene and protein expression. A recent refinement of this process is episcopic imaging, where the autofluorescence of each histology section can be photographed and combined computationally to generate high resolution 3D volume datasets (typically $1-2 \mu \mathrm{m}$ isotropic resolution) [127, 128]. However, these histological approaches are time-consuming and do not lend themselves readily to rapid screening as only one embryo may be imaged at a time. Non-invasive imaging methods, such as MRI, have the potential to offer large time advantages and are increasingly used as an adjunct to histology in the examination of embryo phenotype. This is particularly important considering the large number of embryos required to characterise a phenotype.

\subsection{Non-Destructive Imaging in Ex Vivo Embryo Phenotyping}

Due to the generally low-volumes of embryos that may be phenotyped with conventional histology and its destructive effects on tissue morphology, there is interest in utilising non-invasive imaging methods for morphological screening. Microscopic MRI ( $\mu \mathrm{MRI})$ is one such imaging method, allowing assessments of both embryo development [129] and phenotype [130].

The use of high-resolution $\mu \mathrm{MRI}$ for cardiovascular phenotyping was initiated in the 1990s by Smith and colleagues [131], where they developed a technique to image embryo vasculature. Later, Jacobs and colleagues produced high resolution MR images of single embryos [132], with the goal of creating 3D anatomical atlases as an advance over the $2 \mathrm{D}$ histology-based atlases [126] commonly used, although with a long scan time per embryo. This work has recently been extended by Petiet et al. who produced a database of embryonic, neonatal and adult mice imaged at a number of developmental time points using their method to characterise heart phenotypes in mid-gestation embryos [129, 130].

\section{3. $\mu$ MRI of Multiple Embryos}

Techniques have also been developed to image multiple embryos, in a single acquisition [133]. This method has recently been optimised for cardiac phenotyping in developing embryos [134] and can allow for up to 40 embryos to be imaged in a single 10-hour scan, through the use of a customised imaging coil. In this method, embryos are dissected from the mother and fixed for at least two weeks in a solution of formaldehyde and an MR contrast agent, such as Gd-DTPA. The use of these agents as so-called active stains for ex vivo tissue [135], boosts SNR and enhances image quality when performing $\mu \mathrm{MRI}$. This SNR improvement is important for embryo imaging as their small size requires very high resolution datasets $(<\sim 50 \mu \mathrm{m}$ isotropic), which can take days to acquire without active staining. As the agent is given sufficient time to penetrate fully into embryo tissues, tissue $T_{1}$ relaxation time is reduced, allowing shorter scan-times to be used without affecting SNR and thus image quality. An isotropic 3D dataset is then acquired that may be sliced arbitrarily and viewed in any direction. An outline of the imaging process is shown in Fig. (11). The detailed heart anatomy seen in typical MR images is shown in Fig. (12).

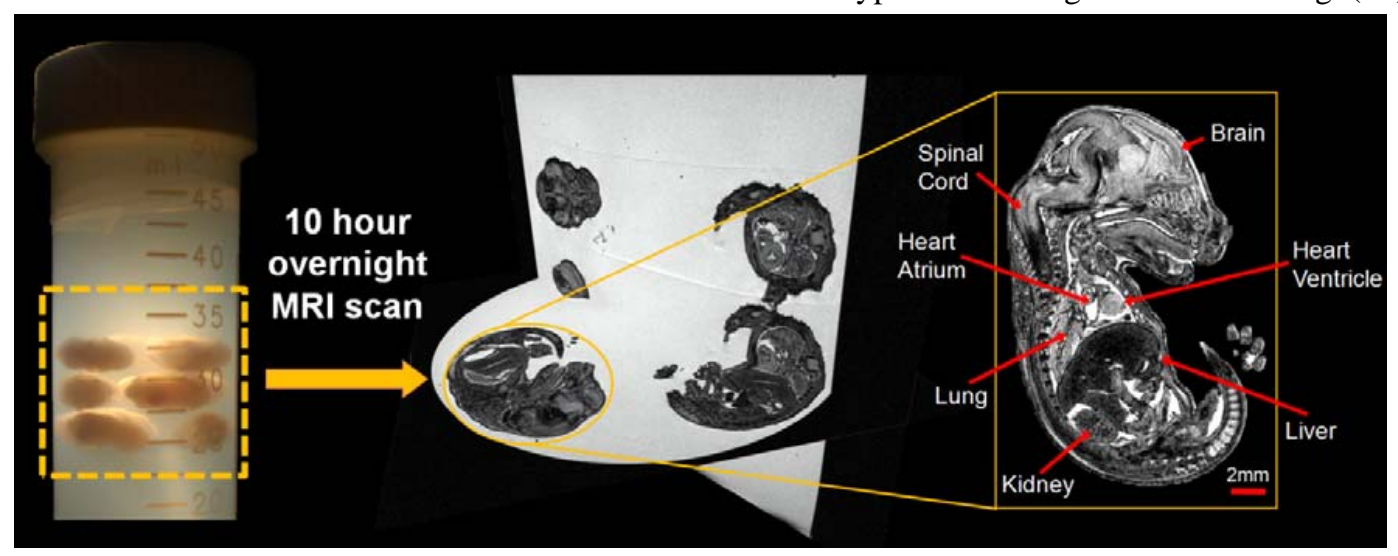

Fig. (11). Outline of the high-throughput embryo imaging process. From left to right: multiple embryos can be embedded into a centrifuge tube and imaged overnight $(52 \mu \mathrm{m}$ isotropic resolution). A 3D volume is then produced which can then be viewed at any angle. A sagittal slice from a single embryo shows the quality of anatomical detail in resulting images. 


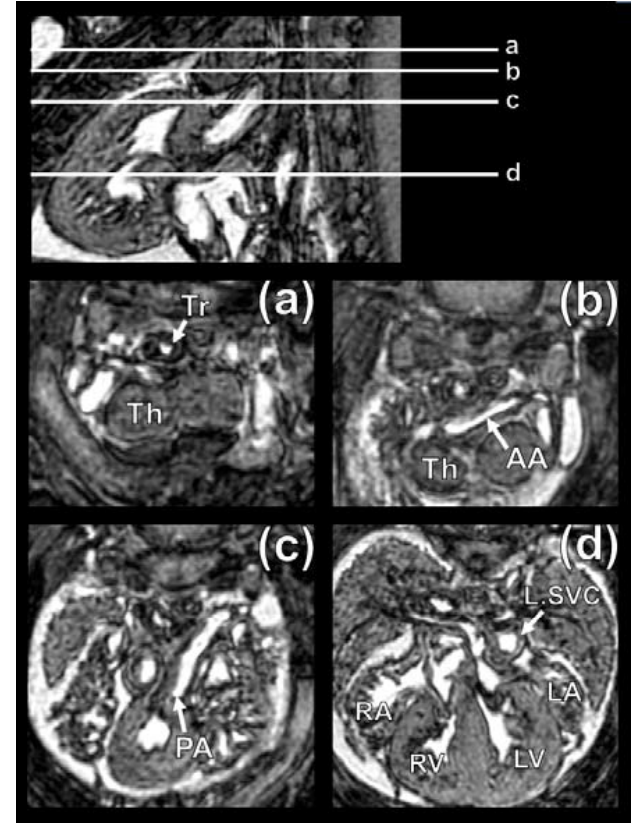

Fig. (12). Normal mouse embryo heart anatomy at 15.5 days post coitum (dpc) as seen by $\mu$ MRI. a-d show successive axial slices through the thorax at the levels indicated. Key: Tr=Trachea, $\mathrm{Th}=$ Thalamus, $\mathrm{AA}=\mathrm{Ascending}$ Aorta, $\mathrm{PA}=$ Pulmonary Artery, L.SVC=Left Superior Vena Cava, LA=Left Atrium, RA=Right Atrium, LV=Left Ventricle, RV=Right Ventricle. Note: dimension of heart is approximately $2 \mathrm{~mm}$ in diameter.

\subsection{Phenotyping of the Chd7 Model of Charge Syndrome}

Cardiac phenotypes can be identified with the multi embryo $\mu$ MRI technique. One example is screening of $C h d 7$ heterozygous embryos [134]. CHD7 is a transcription factor implicated in CHARGE syndrome [136] - a condition with an incidence of 1 in 10,000, partly characterised by defects the cardiovascular system. Ventricular septal defects were identified using MRI in heterozygous mice (Fig. 13), as seen in axial images and volume rendering; great vessels are also readily visible allowing phenotypes in these regions to be identified.

\subsection{Future Direction of Embryo Phenotyping Using MRI}

In vivo imaging in utero with MRI has been reported $[137,138]$ and is able to show structural images of embryos within the mother. However, the continuous foetal motion requires relatively short scans to be taken to reduce move- ment artefacts in the image. This places a limit on the achievable resolution (typically $\sim 150$ um in-plane), signal and contrast to noise. An additional difficulty for the assessment of cardiac function in embryos has been the synchronisation of the image acquisition with the cardiac cycle. Recent developments have enabled the acquisition of 3D cine datasets of the embryo heart in vivo [139]. Manganese, which is increasingly used as an MRI contrast agent in preclinical brain studies for activation and tract-tracing applications, has been applied to the embryo [140]. Its use, in conjunction with in utero MRI, could offer functional information to future embryo heart studies.

A full comprehensive analysis of cardiac phenotype and development in embryos will continue to need a complementary set of imaging modalities ranging from optical microscopy to MRI and ultrasound. The strength of MRI, currently, is its ability to rapidly create non-invasive $3 \mathrm{D}$ datasets of multiple embryos without distortion and with good delineation of soft tissue and vascular anatomy. However, the traditional strength of MRI has been its capability to perform longitudinal studies, where changes in an individual animal may be followed over time. With advances in in utero techniques it may soon be possible to carry out a temporal characterisation of heart development from embryo to adult.

\section{CONCLUSION}

The challenge for cardiac imaging is the accurate assessment of cardiac structure and function in a non-invasive fashion with high temporal and spatial resolution. Ideally, techniques should be widely available, suited to both experimental and clinical conditions, and applicable to a variety of physiological and pathological states. Cardiac MRI techniques have become important tools in the research environment with a steadily increasing number of research facilities implementing CMR.

It is clear that these imaging techniques provide a broad range of measures of cardiac integrity that are related to the functional status of the tissue. One of the major advantages of CMR is its non-invasive nature which lends itself to repeated assessment of cardiac function in the same animal. This allows the tracking of pathological changes over time and as the same animal is used repeatedly increases the statistical power to detect differences. This unique information, in the form of high resolution structural and functional images of the heart, may lead to better understanding of cardiac pathologies and potential treatment strategies. Additionally, using techniques from the clinical setting should help to im-
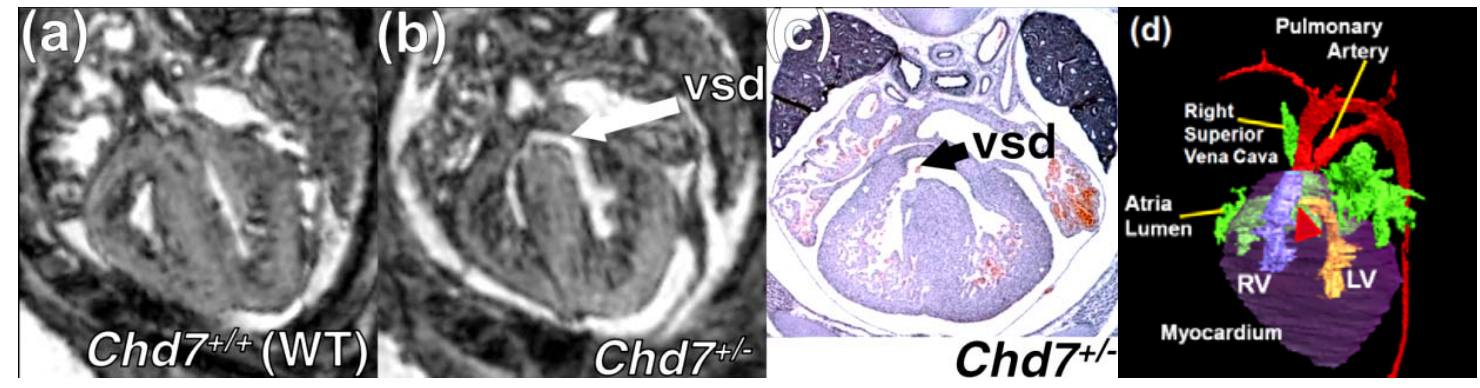

Fig. (13). Axial slices through $C h d 7^{+/+}$and $C h d 7^{+/}$embryos as part of a high throughput MRI screen. (a) Image showing a wild-type embryo with an intact ventricular septum. (b) image from an $C h d 7^{+/-}$embryo showing a ventricular septal defect (VSD) which was confirmed on later H\&E histology (c) [134]. Volume rendering (d) of the same $C h d 7^{+/}$heart and great vessels allows visualisation of the VSD (red triangle) in 3 dimensions. 
prove the applicability of experimental findings to human patients.

\section{ACKNOWLEDGMENTS}

This work was supported by the British Heart Foundation, Biotechnology and Biological Sciences Research Council and the Engineering and Physical Sciences Research Council.

\section{ABBREVIATIONS}

$3 \mathrm{D}$

ASL

ATP

BOLD

CMR

DTI

ECG

ED

EDV

$\mathrm{EF}$

ES

ESV

Gd-DTPA

GRE

LAD

LGE

LV

LVM

MEMRI

MI

MR

MRA

MRI

$\mathrm{PCr}$

$\mathrm{Pi}$

RF

SNR

SPAMM

SPIOs

SSFP

SV

TOF

\section{REFERENCES}

[1] Poon M, Fuster V, Fayad Z. Cardiac magnetic resonance imaging: a "one-stop-shop" evaluation of myocardial dysfunction. Curr Opin Cardiol 2002; 17: 663-70.
Boxerman JL, Mosher TJ, McVeigh ER, Atalar E, Lima JA, Bluemke DA. Advanced MR imaging techniques for evaluation of the heart and great vessels. Radiographics 1998; 18: 543-64.

Ruff J, Wiesmann F, Hiller KH, et al. Magnetic resonance microimaging for noninvasive quantification of myocardial function and mass in the mouse. Magn Reson Med 1998; 40: 43-8.

Siri FM, Jelicks LA, Leinwand LA, Gardin JM. Gated magnetic resonance imaging of normal and hypertrophied murine hearts. Am J Physiol 1997; 272: H2394-402.

Vallee JP, Ivancevic MK, Nguyen D, Morel DR, Jaconi M. Current status of cardiac MRI in small animals. MAGMA 2004; 17: 14956.

Schepkin VD, Brey WW, Gor'kov PL, Grant SC. Initial in vivo rodent sodium and proton MR imaging at 21.1 T. Magn Reson Imaging 2009 [Epub ahead of print].

Gilson WD, Kraitchman DL. Cardiac magnetic resonance imaging in small rodents using clinical $1.5 \mathrm{~T}$ and $3.0 \mathrm{~T}$ scanners. Methods 2007; 43: 35-45.

Cassidy PJ, Schneider JE, Grieve SM, Lygate C, Neubauer S, Clarke K. Assessment of motion gating strategies for mouse magnetic resonance at high magnetic fields. J Magn Reson Imaging 2004; 19: 229-37.

Sekihara K. Steady-state magnetizations in rapid NMR imaging using small flip angles and short repetition intervals. IEEE Trans Med Imaging 1987; 6: 157-64.

Geest RJ, Buller VG, Jansen E, et al. Comparison between manual and semiautomated analysis of left ventricular volume parameters from short-axis MR images. J Comput Assist Tomogr 1997; 21: 756-65.

Heijman E, Aben JP, Penners C, et al. Evaluation of manual and automatic segmentation of the mouse heart from CINE MR images. J Magn Reson Imaging 2008; 27: 86-93.

Furber A, Balzer P, Cavaro-Menard C, et al. Experimental validation of an automated edge-detection method for a simultaneous determination of the endocardial and epicardial borders in short-axis cardiac MR images: application in normal volunteers. J Magn Reson Imaging 1998; 8: 1006-14.

Young AA, Cowan BR, Thrupp SF, Hedley WJ, Dell'Italia LJ. Left ventricular mass and volume: fast calculation with guide-point modeling on MR images. Radiology 2000; 216: 597-602.

Heiberg E, Wigström L, Carlsson M, Bolger AF, Karlsson M. Time resolved three-dimensional automated segmentation of the left ventricle. Proc IEEE Comp Cardiol 2005; 32: 599-602.

Slawson SE, Roman BB, Williams DS, Koretsky AP. Cardiac MRI of the normal and hypertrophied mouse heart. Mag Reson Med 1998; 39: 980-7.

ten Hove M, Lygate CA, Fischer A, et al. Reduced inotropic reserve and increased susceptibility to cardiac ischemia/reperfusion injury in phosphocreatine-deficient guanidinoacetate-N-methyltransferase-knockout mice. Circulation 2005; 111: 2477-85.

Alfakih K, Reid S, Jones T, Sivananthan M. Assessment of ventricular function and mass by cardiac magnetic resonance imaging. Eur Radiol 2004; 14: 1813-22.

Gutberlet M, Abdul-Khaliq H, Grothoff M, et al. Evaluation of left ventricular volumes in patients with congenital heart disease and abnormal left ventricular geometry. Rofo 2003; 175: 942-51.

Germain P, Roul G, Kastler B, Mossard JM, Bareiss P, Sacrez A. Inter-study variability in left ventricular mass measurement. Comparison between M-mode echography and MRI. Eur Heart J 1992; 13: 1011-9.

Streif JU, Herold V, Szimtenings M, et al. In vivo time-resolved quantitative motion mapping of the murine myocardium with phase contrast MRI. Magn Reson Med 2003; 49: 315-21.

Zerhouni EA, Parish DM, Rogers WJ, Yang A, Shapiro EP. Human heart: tagging with MR imaging -- a method for noninvasive assessment of myocardial motion. Radiology 1988; 169: 5963.

Axel L, Dougherty L. MR imaging of motion with spatial modulation of magnetization. Radiology 1989; 171: 841-5.

Zhou R, Pickup S, Glickson JD, Scott CH, Ferrari VA. Assessment of global and regional myocardial function in the mouse using cine and tagged MRI. Magn Reson Med 2003; 49: 760-4.

McVeigh E. Regional myocardial function. Cardiol Clin 1998; 16 : 189-206. 
[25] Atalar E, McVeigh ER. Optimization of tag thickness for measuring position with magnetic resonance imaging. IEEE Trans Med Imaging 1994; 13: 152-60.

[26] Guttman MA, Prince JL, McVeigh ER. Tag and contour detection in tagged MR images of the left ventricle. IEEE Trans Med Imaging 1994; 13: 74-88.

[27] Liu W, Chen JJ, Ji SB, et al. Harmonic phase MR tagging for direct quantification of Lagrangian strain in rat hearts after myocardial infarction. Mag Reson Med 2004; 52: 1282-90.

[28] Aletras AH, Ding S, Balaban RS, Wen H. DENSE: displacement encoding with stimulated echoes in cardiac functional MRI. J Magn Reson 1999; 137: 247-52.

[29] Osman NF, Sampath S, Atalar E, Prince JL. Imaging longitudinal cardiac strain on short-axis images using strain-encoded MRI. Magn Reson Med 2001; 46: 324-34.

[30] Kraitchman DL, Sampath S, Castillo E, et al. Quantitative ischemia detection during cardiac magnetic resonance stress testing by use of FastHARP. Circulation 2003; 107: 2025-30.

[31] Osman NF, Kerwin WS, McVeigh ER, Prince JL. Cardiac motion tracking using CINE harmonic phase (HARP) magnetic resonance imaging. Mag Reson Med 1999; 42: 1048-60.

[32] Yeon SB, Reichek N, Tallant BA, et al. Validation of in vivo myocardial strain measurement by magnetic resonance tagging with sonomicrometry. J Am Coll Cardiol 2001; 38: 555-61.

[33] Moore CC, McVeigh ER, Zerhouni EA. Noninvasive measurement of three-dimensional myocardial deformation with tagged magnetic resonance imaging during graded local ischemia. J Cardiovasc Magn Reson 1999; 1: 207-22.

[34] Henson RE, Song SK, Pastorek JS, Ackerman JJH, Lorenz CH. Left ventricular torsion is equal in mice and humans. Am J Physiol Heart Circ Physiol 2000; 278: H1117-23.

[35] Zhang H, Shea SM, Park V, et al. Accurate myocardial T1 measurements: toward quantification of myocardial blood flow with arterial spin labeling. Magn Reson Med 2005; 53: 1135-42.

[36] Barkhausen J, Hunold P, Jochims M, Debatin JF. Imaging of myocardial perfusion with magnetic resonance. J Magn Reson Imaging 2004; 19: 750-7.

[37] Arai AE. Magnetic resonance first-pass myocardial perfusion imaging. Top Magn Reson Imaging 2000; 11: 383-98.

[38] Pickup S, Zhou R, Glickson J. MRI estimation of the arterial input function in mice. Acad Radiol 2003; 10: 963-8.

[39] Belle V, Kahler E, Waller C, et al. In vivo quantitative mapping of cardiac perfusion in rats using a noninvasive MR spin-labeling method. J Magn Reson Imaging 1998; 8: 1240-5.

[40] Kober F, Iltis I, Izquierdo M, et al. High-resolution myocardial perfusion mapping in small animals in vivo by spin-labeling gradient-echo imaging. Magn Reson Med 2004; 51: 62-7.

[41] Streif JU, Nahrendorf M, Hiller KH, et al. In vivo assessment of absolute perfusion and intracapillary blood volume in the murine myocardium by spin labeling magnetic resonance imaging. Magn Reson Med 2005; 53: 584-92.

[42] Waller C, Kahler E, Hiller KH, et al. Myocardial perfusion and intracapillary blood volume in rats at rest and with coronary dilatation: MR imaging in vivo with use of a spin-labeling technique. Radiology 2000; 215: 189-97.

[43] Waller C, Hiller KH, Voll S, Haase A, Ertl G, Bauer WR. Myocardial perfusion imaging using a non-contrast agent MR imaging technique. Int J Cardiovasc Imaging 2001; 17: 123-32.

[44] Vandsburger MH, French BA, Helm PA, et al. Multi-parameter in vivo cardiac magnetic resonance imaging demonstrates normal perfusion reserve despite severely attenuated beta-adrenergic functional response in neuronal nitric oxide synthase knockout mice. Eur Heart J 2007; 28: 2792-8.

[45] Iltis I, Kober F, Dalmasso C, Lan C, Cozzone PJ, Bernard M. In vivo assessment of myocardial blood flow in rat heart using magnetic resonance imaging: effect of anesthesia. J Magn Reson Imaging 2005; 22: 242-7.

[46] Kober F, Iltis I, Cozzone PJ, Bernard M. Myocardial blood flow mapping in mice using high-resolution spin labeling magnetic resonance imaging: influence of ketamine/xylazine and isoflurane anesthesia. Magn Reson Med 2005; 53: 601-6.

[47] Atalay MK, Forder JR, Chacko VP, Kawamoto S, Zerhouni EA. Oxygenation in the rabbit myocardium: assessment with susceptibility-dependent MR imaging. Radiology 1993; 189: 759-64.

[48] Bandettini PA, Wong EC, Jesmanowicz A, Hinks RS, Hyde JS. Spin-Echo and gradient-echo EPI of human brain activation using
BOLD contrast: a comparative study at 1.5 T. NMR Biomed 1994; 7: 12-20.

[49] Shea SM, Fieno DS, Schirf BE, et al. T2-prepared steady-state free precession blood oxygen level-dependent MR imaging of myocardial perfusion in a dog stenosis model. Radiology 2005; 236: 5039.

[50] Kaandorp TAM, Lamb HJ, van der Wall EE, de Roos A, Bax JJ. Cardiovascular MR to access myocardial viability in chronic ischaemic LV dysfunction. Heart 2005; 91: 1359-65.

[51] Wiesmann F, Ruff J, Engelhardt S, et al. Dobutamine-stress magnetic resonance microimaging in mice: acute changes of cardiac geometry and function in normal and failing murine hearts. Circ Res 2001; 88: 563-9.

[52] Nahrendorf M, Wiesmann F, Hiller KH, et al. Serial cine-magnetic resonance imaging of left ventricular remodeling after myocardial infarction in rats. J Magn Reson Imaging 2001; 14: 547-55.

[53] Kuijpers D, Ho KYJA, van Dijkman PRM, Vliegenthart R, Oudkerk M. Dobutamine cardiovascular magnetic resonance for the detection of myocardial ischemia with the use of myocardial tagging. Circulation 2003; 107: 1592-7.

[54] Kramer CM, Malkowski MJ, Mankad S, Theobald TM, Pakstis DL, Rogers WJ. Magnetic resonance tagging and echocardiographic response to dobutamine and functional improvement after reperfused myocardial infarction. Am Heart J 2002; 143: 1046-51.

[55] Williams SP, Gerber HP, Giordano FJ, et al. Dobutamine stress cine-MRI of cardiac function in the hearts of adult cardiomyocytespecific VEGF knockout mice. J Magn Reson Imaging 2001; 14: 374-82.

[56] Wu KC, Lima JAC. Noninvasive Imaging of myocardial viability Current techniques and future developments. Circ Res 2003; 93: 1146-58.

[57] Kim RJ, Fieno DS, Parrish TB, et al. Relationship of MRI delayed contrast enhancement to irreversible injury, infarct age, and contractile function. Circulation 1999; 100: 1992-2002.

[58] Mcnamara MT, Tscholakoff D, Revel D, et al. Differentiation of reversible and irreversible myocardial injury by MR imaging with and without Gadolinium-DTPA. Radiology 1986; 158: 765-9.

[59] Rehwald WG, Fieno DS, Chen EL, Kim RJ, Judd RM. Myocardial magnetic resonance imaging contrast agent concentrations after reversible and irreversible ischemic injury. Circulation 2002; 105: 224-9.

[60] Yang ZQ, Berr SS, Gilson WD, Toufektsian MC, French BA. Simultaneous evaluation of infarct size and cardiac function in intact mice by contrast-enhanced cardiac magnetic resonance imaging reveals contractile dysfunction in noninfarcted regions early after myocardial infarction. Circulation 2004; 109: 1161-7.

[61] Price AN, Cheung KK, Lim SL, Hausenloy DJ, Yellon DM, Lythgoe MF. Late gadolinium enhanced MRI in small animal models of myocardial infarction. J Cardiovasc Magn Reson 2010; 12: $\mathrm{P} 98$

[62] Hu TCC, Pautler RG, MacGowan GA, Koretsky AP. Manganeseenhanced MRI of mouse heart during changes in inotropy. Magn Reson Med 2001; 46: 884-90.

[63] Reese T, Bochelen D, Sauter A, Beckmann N, Rudin M. Magnetic resonance angiography of the rat cerebrovascular system without the use of contrast agents. NMR Biomed 1999; 12: 189-96.

[64] Beckmann N. High resolution magnetic resonance angiography non-invasively reveals mouse strain differences in the cerebrovascular anatomy in vivo. Mag Reson Med 2000; 44: 252-8.

[65] Beckmann N, Stirnimann R, Bochelen D. High-resolution magnetic resonance angiography of the mouse brain: application to murine focal cerebral ischemia models. J Magn Reson 1999; 140: 442-50.

[66] Ruff J, Wiesmann F, Lanz T, Haase A. Magnetic resonance imaging of coronary arteries and heart valves in a living mouse: techniques and preliminary results. J Magn Reson 2000; 146: 2906.

[67] Miraux S, Serres S, Thiaudiere E, Canioni P, Merle M, Franconi JM. Gadolinium-enhanced small-animal TOF magnetic resonance angiography. MAGMA 2004; 17: 348-52.

[68] Ohlstein EH, Romanic AM, Clark LV, et al. Application of in vivo and ex vivo magnetic resonance imaging for evaluation of tranilast on neointima formation following balloon angioplasty of the rat carotid artery. Cardiovasc Res 2000; 47: 759-68.

[69] Chandra S, Clark LV, Coatney RW, Phan L, Sarkar SK, Ohlstein EH. Application of serial in vivo magnetic resonance imaging to evaluate the efficacy of endothelin receptor antagonist SB 217242 
in the rat carotid artery model of neointima formation. Circulation 1998; 97: 2252-8.

[70] Choudhury RP, Fuster V, Badimon JJ, Fisher EA, Fayad ZA. MRI and characterization of atherosclerotic plaque: emerging applications and molecular Imaging. Arterioscler Thromb Vasc Biol 2002; 22: 1065-74.

[71] Manka DR, Gilson W, Sarembock I, Ley K, Berr SS. Noninvasive in vivo magnetic resonance imaging of injury-induced neointima formation in the carotid artery of the apolipoprotein-E null mouse. J Magn Reson Imaging 2000; 12: 790-4.

[72] Hockings PD, Roberts T, Galloway GJ, et al. Repeated threedimensional magnetic resonance imaging of atherosclerosis development in innominate arteries of low-density lipoprotein receptorknockout mice. Circulation 2002; 106: 1716-21.

[73] Itskovich VV, Lieb M, Aguinaldo JGS, Samber DD, Ramirez F, Fayad ZA. Magnetic resonance microscopy quantifies the disease progression in Marfan syndrome mice. J Magn Reson Imaging 2003; 17: 435-9.

[74] Itskovich VV, Choudhury RP, Aguinaldo JGS, et al. Characterization of aortic root atherosclerosis in ApoE knockout mice: Highresolution in vivo and ex vivo MRM with histological correlation. Mag Reson Med 2003; 49: 381-5.

[75] McAteer MA, Sibson NR, von Zur MC, et al. In vivo magnetic resonance imaging of acute brain inflammation using microparticles of iron oxide. Nat Med 2007; 13: 1253-8.

[76] Holmes AA, Scollan DF, Winslow RL. Direct histological validation of diffusion tensor MRI in formaldehyde-fixed myocardium. Mag Reson Med 2000; 44: 157-61.

[77] Hsu EW, Muzikant AL, Matulevicius SA, Penland RC, Henriquez CS. Magnetic resonance myocardial fiber-orientation mapping with direct histological correlation. Am J Physiol 1998; 43: H1627-34.

[78] Scollan DF, Holmes A, Winslow R, Forder J. Histological validation of myocardial microstructure obtained from diffusion tensor magnetic resonance imaging. Am J Physiol 1998; 44: H2308-18.

[79] Chen JJ, Song SK, Liu W, et al. Remodeling of cardiac fiber structure after infarction in rats quantified with diffusion tensor MRI. Am J Physiol 2003; 285: H946-54.

[80] Chen JJ, Liu W, Zhang HY, et al. Regional ventricular wall thickening reflects changes in cardiac fiber and sheet structure during contraction: quantification with diffusion tensor MRI. Am J Physiol 2005; 289: H1898-1907.

[81] Kohler S, Hiller KH, Waller C, Bauer WR, Haase A, Jakob PM. Investigation of the microstructure of the isolated rat heart: A comparison between $\mathrm{T}^{*} 2$ - and diffusion-weighted MRI. Magn Reson Med 2003; 50: 1144-50.

[82] Jiang Y, Pandya K, Smithies O, Hsu EW. Three-dimensional diffusion tensor microscopy of fixed mouse hearts. Magn Reson Med 2004; 52: 453-60.

[83] Chacko VP, Aresta F, Chacko SM, Weiss RG. MRI/MRS assessment of in vivo murine cardiac metabolism, morphology, and function at physiological heart rates. Am J Physiol 2000; 279: H221824.

[84] Naumova AV, Weiss RG, Chacko VP. Regulation of murine myocardial energy metabolism during adrenergic stress studied by in vivo P-31 NMR spectroscopy. Am J Physiol 2003; 285: H1976-79.

[85] Jansen MA, Van Emous JG, Nederhoff MGJ, Van Echteld CJA. Assessment of myocardial viability by intracellular Na-23 magnetic resonance Imaging. Circulation 2004; 110: 3457-64.

[86] Lewandowski ED. Cardiac carbon 13 magnetic resonance spectroscopy: On the horizon or over the rainbow? J Nucl Cardiol 2002; 9: 419-28.

[87] Herrero P, Gropler RJ. Imaging of myocardial metabolism. J Nucl Cardiol 2005; 12: 345-58.

[88] Verdouw PD, van den Doel MA, de Zeeuw S, Duncker DJ. Animal models in the study of myocardial ischaemia and ischaemic syndromes. Cardiovasc Res 1998; 39: 121-35.

[89] Christian TF, O'Connor MK, Schwartz RS, Gibbons RJ, Ritman EL. Technetium-99m MIBI to assess coronary collateral flow during acute myocardial infarction in two closed-chest animal models. J Nucl Med 1997; 38: 1840-6.

[90] Itter G, Jung W, Juretschke R, Schoelkens BA, Linz W. A model of chronic heart failure in spontaneous hypertensive rats (SHR). Lab Anim 2004; 38: 138-48.

[91] Waller C, Hiller KH, Kahler E, et al. Serial magnetic resonance imaging of microvascular remodeling in the infarcted rat heart. Circulation 2001; 103: 1564-9.
[92] Hu K, Gaudron P, Anders HJ, et al. Chronic effects of early started angiotensin converting enzyme inhibition and angiotensin AT(1)receptor subtype blockade in rats with myocardial infarction: role of bradykinin. Cardiovasc Res 1998; 39: 401-12.

[93] Arai T, Kofidis T, Bulte JWM, et al. Dual in vivo magnetic resonance evaluation of magnetically labeled mouse embryonic stem cells and cardiac function at 1.5 T. Mag Reson Med 2006; 55: 203 9.

[94] Jennings RB, Sommers HM, Smyth GA, Flack HA, Linn H. Myocardial necrosis induced by temporary occlusion of a coronary artery in the dog. Arch Pathol 1960; 70: 68-78.

[95] Lusis AJ. Atherosclerosis. Nature 2000; 407: 233-41.

[96] Narayanaswamy M, Wright KC, Kandarpa K. Animal models for atherosclerosis, restenosis, and endovascular graft research. J Vas Interv Radiol 2000; 11: 5-17.

[97] Zhang SH, Reddick RL, Piedrahita JA, Maeda N. Spontaneous hypercholesterolemia and arterial lesions in mice lacking apolipoprotein E. Science 1992; 258: 468-71.

[98] Gutstein DE, Fuster V. Pathophysiology and clinical significance of atherosclerotic plaque rupture. Cardiovasc Res 1999; 41: 323-33.

[99] Cai JM, Hatsukami TS, Ferguson MS, et al. In vivo quantitative measurement of intact fibrous cap and lipid-rich necrotic core size in atherosclerotic carotid plaque - Comparison of high-resolution, contrast-enhanced magnetic resonance imaging and histology. Circulation 2005; 112: 3437-44.

[100] Douglas SA, Louden C, Vickeryclark LM, et al. A role for endogenous endothelin-1 in neointimal formation after rat carotid-artery balloon angioplasty. Protective effects of the novel nonpeptide endothelin receptor antagonist Sb-209670. Circ Res 1994; 75: 190-7.

[101] Gabeler EE, van Hillegersberg R, Statius van Eps RG, et al. A comparison of balloon injury models of endovascular lesions in rat arteries. BMC Cardiovasc Disord 2002; 2: 16-27.

[102] Summers RM, Hedlund LW, Cofer GP, Gottsman MB, Manibo JF, Johnson GA. MR microscopy of the rat carotid-artery after balloon injury by using an implanted imaging coil. Mag Reson Med 1995; 33: 785-9.

[103] Yamamoto T, Ikuta K, Oi K, et al. In vivo MR detection of vascular endothelial injury using a new class of MRI contrast agent. Bioorg Med Chem Lett 2004; 14: 2787-90.

[104] Gnecchi M, He H, Liang OD, et al. Paracrine action accounts for marked protection of ischemic heart by Akt-modified mesenchymal stem cells. Nat Med 2005; 11: 367-8.

[105] Wisenberg G, Lekx K, Zabel P, et al. Cell tracking and therapy evaluation of bone marrow monocytes and stromal cells using SPECT and CMR in a canine model of myocardial infarction. $J$ Cardiovasc Magn Reson 2009; 11: 11.

[106] Carr CA, Stuckey DJ, Tatton L, et al. Bone marrow-derived stromal cells home to and remain in the infarcted rat heart but fail to improve function: an in vivo cine-MRI study. Am J Physiol Heart Circ Physiol 2008; 295: H533-42.

[107] Daniel JS, Carolyn AC, Enca M, et al. Iron particles for noninvasive monitoring of bone marrow stromal cell engraftment into, and isolation of viable engrafted donor cells from, the heart. Stem Cells 2006; 24: 1968-75.

[108] Engelmann MG, Theiss HD, Hennig-Theiss C, et al. Autologous bone marrow stem cell mobilization induced by granulocyte colony-stimulating factor after subacute ST-segment elevation myocardial infarction undergoing late revascularization: final results from the G-CSF-STEMI (Granulocyte Colony-Stimulating Factor ST-Segment Elevation Myocardial Infarction) Trial. J Am Coll Cardiol 2006; 48: 1712-21.

[109] Kim YJ, Kuh YM, Choe KO, et al. In vivo magnetic resonance imaging of injected mesenchymal stem cells in rat myocardial infarction; simultaneous cell tracking and left ventricular function measurement. Int J Cardiovasc Imaging 2009; 25: 99-109.

[110] Moelker AD, Baks T, van den Bos EJ, et al. Reduction in infarct size, but no functional improvement after bone marrow cell administration in a porcine model of reperfused myocardial infarction. Eur Heart J 2006; 27: 3057-64.

[111] van Laake LW, Passier R, Doevendans PA, Mummery CL. Human embryonic stem cell-derived cardiomyocytes and cardiac repair in rodents. Circ Res 2008; 102: 1008-10.

[112] Forrester JS, White AJ, Matsushita S, Chakravarty T, Makkar RR. New paradigms of myocardial regeneration post-infarction: tissue preservation, cell environment, and pluripotent cell sources. JACC: Cardiovasc Interv 2009; 2 : 1-8. 
[113] Janssens S, Dubois C, Bogaert J, et al. Autologous bone marrowderived stem-cell transfer in patients with ST-segment elevation myocardial infarction: double-blind, randomised controlled trial. Lancet 2006; 367: 113-21.

[114] Wollert KC, Meyer GP, Lotz J, et al. Intracoronary autologous bone-marrow cell transfer after myocardial infarction: the BOOST randomised controlled clinical trial. Lancet 2004; 364: 141-8.

[115] Amsalem Y, Mardor Y, Feinberg MS, et al. Iron-oxide labeling and outcome of transplanted mesenchymal stem cells in the infarcted myocardium. Circulation 2007; 116: I38-45.

[116] Chris Heyn JAR. In vivo magnetic resonance imaging of single cells in mouse brain with optical validation. Magn Reson Med 2006; 55: 23-9.

[117] Rogers WJ, Meyer CH, Kramer CM. Technology insight: in vivo cell tracking by use of MRI. Nat Clin Pract Cardiovasc Med 2006; 3: 554-62.

[118] Shapiro EM, Skrtic S, Sharer K, Hill JM, Dunbar CE, Koretsky AP. MRI detection of single particles for cellular imaging. Proc Nat Acad Sci U S A 2004; 101: 10901-6.

[119] Wood JC, Otto-Duessel M, Aguilar M, et al. Cardiac iron determines cardiac $\mathrm{T} 2 *, \mathrm{~T} 2$, and $\mathrm{T} 1$ in the gerbil model of iron cardiomyopathy. Circulation 2005; 112: 535-43.

[120] Wilhelm C, Gazeau F. Universal cell labelling with anionic magnetic nanoparticles. Biomaterials 2008; 29: 3161-74.

[121] Neri M, Maderna C, Cavazzin C, et al. Efficient in vitro labeling of human neural precursor cells with superparamagnetic iron oxide particles: relevance for in vivo cell tracking. Stem Cells 2008; 26: 505-16.

[122] Farrell E, Wielopolski P, Pavljasevic P, et al. Effects of iron oxide incorporation for long term cell tracking on MSC differentiation in vitro and in vivo. Biochem Biophys Res Commun 2008; 369: 107681 .

[123] Paigen K. One hundred years of mouse genetics: an intellectual history. I. The classical period (1902-1980). Genetics 2003; 163: 17 .

[124] Mouse Genome Sequencing Consortium. Chinwalla AT, Cook LL, Delehaunty KD, et al. Initial sequencing and comparative analysis of the mouse genome. Nature 2002; 420: 520-62.

[125] The International Mouse Knockout Consortium. Collins FS, Rossant J, Wurst W. A Mouse for All Reasons. Cell 2007; 128: 9-13.

[126] Kaufman MH. The atlas of mouse development. London: Academic Press; 1992.

[127] Weninger WJ, Geyer SH, Mohun TJ, et al. High-resolution episcopic microscopy: a rapid technique for high detailed 3D analysis of gene activity in the context of tissue architecture and morphology. Anat Embryol 2006; 211: 213-21.

[128] Weninger WJ, Mohun TJ. Phenotyping transgenic embryos: a rapid $3-D$ screening method based on episcopic fluorescence image capturing. Nat Genet 2002; 30: 59-65.

[129] Petiet AE, Kaufman MH, Goddeeris MM, Brandenburg J, Elmore SA, Johnson GA. High-resolution magnetic resonance histology of the embryonic and neonatal mouse: A 4D atlas and morphologic database. Proc Natl Acad Sci U S A 2008; 105: 12331-6.

[130] Goddeeris MM, Rho S, Petiet A, et al. Intracardiac septation requires hedgehog-dependent cellular contributions from outside the heart. Development 2008; 135: 1887-95.

[131] Smith BR, Johnson GA, Groman EV, Linney E. Magnetic resonance microscopy of mouse embryos. Proc Natl Acad Sci U S A 1994; 91: 3530-3.

[132] Jacobs RE, Ahrens ET, Dickinson ME, Laidlaw D. Towards a microMRI atlas of mouse development. Comput Med Imaging Graph 1999; 23: 15-24.

[133] Schneider JE, Bamforth SD, Grieve SM, Clarke K, Bhattacharya S, Neubauer S. High-resolution, high-throughput magnetic paragraph sign resonance imaging of mouse embryonic paragraph sign anatomy using a fast gradient-echo sequence. MAGMA 2003; 16 : 43-51.

[134] Cleary JO, Price AN, Thomas DL, et al. Cardiac phenotyping in ex vivo murine embryos using microMRI. NMR Biomed 2009; 22 857-66.

[135] Johnson GA, Cofer GP, Gewalt SL, Hedlund LW. Morphologic Phenotyping with MR microscopy: the visible mouse. Radiology 2002; 222: 789-93.

[136] Bosman EA, Penn AC, Ambrose JC, Kettleborough R, Stemple DL, Steel KP. Multiple mutations in mouse Chd7 provide models for CHARGE syndrome. Hum Mol Genet 2005; 14: 3463-76.

[137] Chapon C, Franconi F, Roux J, Marescaux L, Le Jeune J, Lemaire L. In utero time-course assessment of mouse embryo developmen using high resolution magnetic resonance imaging. Anat Embryol 2002; 206: 131-7.

[138] Hogers B, Gross D, Lehmann V, et al. Magnetic resonance microscopy of mouse embryos in utero. Anat Rec 2000; 260: 373-7.

[139] Nieman BJ, Szulc KU, Turnbull DH. Three-dimensional, in vivo MRI with self-gating and image coregistration in the mouse. Magn Reson Med 2009; 61: 1148-57.

[140] Deans AE, Wadghiri YZ, Berrios-Otero CA, Turnbull DH. Mn enhancement and respiratory gating for in utero MRI of the embryonic mouse central nervous system. Magn Reson Med 2008; 59 ; $1320-8$. 